\title{
Uma Plataforma para Cidades Inteligentes baseada na Internet das Coisas
}

\author{
Valter Henrique de Souza Ferreira da Silva ${ }^{1}$, Alexandre Alvaro ${ }^{1}$ \\ ${ }^{1}$ Departamento de Computação (DComp) - Universidade Federal de São Carlos \\ (UFSCar) - Campus Sorocaba \\ Sorocaba - SP - Brasil \\ valter.bcc@gmail.com, alvaro@ufscar.br
}

\begin{abstract}
Nowadays ICT (Information and Communication Technology) are applied to solve the main common issues that cities all over the world are dealing with, such as transportation, public safety, energy, healthcare, and education, etc. The junction with the technology infrastructure and services of a city makes it gradually a Smart City. These emerging technologies, the Internet and an array of sensors that transform objects of day-to-day into smart objects, enabling the Internet of Things to assist in the process that cities becoming intelligent. Thought the Internet of Things, the smart cities are becoming more viable. So, this paper presents a platform to assist in this direction.
\end{abstract}

Resumo. Hoje em dia a TIC é aplicada visando resolver os principais problemas comuns que as cidades em todo o mundo estão lidando, tais como transporte, segurança pública, energia, saúde e educação, etc. A junção de tecnologia com as infra-estruturas e serviços de uma cidade a transforma gradativamente em uma Cidade Inteligente. Estas tecnologias emergentes, a Internet e uma gama de sensores que transformam os objetos do dia-a-dia em objetos inteligentes, permitem que a Internet das Coisas auxilie no processo de inteligência das cidades. Assim, a Internet das Coisas vem para tornar as Cidades Inteligentes mais viáveis. Neste sentido, este artigo apresenta uma proposta de plataforma para auxiliar nesta direção.

\section{Introdução}

O desenvolvimento de uma cidade é um processo complicado e o governo da cidade enfrenta inúmeros desafios em várias questões frente a esse crescimento, muitas vezes desordenado. No entanto, tornam-se elementos essenciais para a construção de uma cidade competitiva o desenvolvimento de infra-estrutura básica, bem como tecnologias, aplicações e serviços de TICs (Tecnologia da Informação e Comunicação) [Pan 2011]. Neste sentido, para construção de ambientes mais competitivos, as cidades devem concentrar principalmente na aplicação de TICs de última geração em todas as esferas, realizando a incorporação de sensores em equipamentos para hospitais, em redes de energia, ferrovias, pontes, túneis, estradas, edifícios, sistemas de água, barragens, oleodutos e gasodutos e outros objetos em várias partes do mundo, formando a "Internet das Coisas" [Ahson 2008]. Com isso, pode-se integrar a Internet das Coisas através da Cloud Computing [Vaquero 2009] para prover os serviços de forma escalável e eficiente. Neste caso, as pessoas podem gerir a produção e a vida de uma forma mais 
meticulosa e dinâmica, alcançando o estado de inteligência global, e, finalmente, chegar a "Internet das Coisas + Internet = Cidade inteligente" [Kehua 2011].

A Internet das Coisas, embora seja um conceito relativamente novo, é resultado de um longo processo de maturação. Do ponto de vista tecnológico, ela surgiu como uma sucessão de quatro passos. Primeiro, o sucesso da adoção do código de barras e os desenvolvimentos posteriores em torno desse conceito; segundo, a extensão do RFID (Radio Frequency Identification) [Ahson 2008] para além da mera "etiquetagem” de objetos, criando um ambiente inteligente; terceiro, com a introdução conceitual da rede de sensores; e, finalmente, a "emersão" de um universo de objetos autômatos como um mundo à parte.

Por outro lado, o conceito de cidades inteligentes é definido como o uso de TICs para verificar, analisar e integrar as informações-chaves de sistemas centrais nas cidades em execução. Ao mesmo tempo, a cidade inteligente pode obter uma resposta inteligente a diferentes tipos de necessidades, incluindo o dia a dia da população, proteção ambiental, segurança e serviços públicos da cidade, atividades industriais e comerciais [Kehua 2011].

Com isso, este artigo apresenta uma plataforma para cidades inteligentes baseada na internet das coisas. A seção a seguir apresenta conceitos importantes sobre estes 2 tópicos supracitados; a seção 3 apresenta o framework proposto; a seção 4 mostra como pode ser útil o framework citando alguns exemplos; e finalmente a seção 5 apresenta as conclusões e perspectivas de trabalhos futuros.

\section{Internet das Coisas e Cidades Inteligentes}

Desde 2000, os cientistas do MIT, do grupo Auto-ID Labs, visionavam o futuro da tecnologia RFID [Sarma 2000]: "um mundo no qual todos os dispositivos eletrônicos estão em rede e cada objeto, seja objetos físicos (animais ou seres humanos) ou eletrônico, será eletronicamente etiquetados com informações pertinentes a estes objetos. Nós encaramos o uso de tags físicas que permitam o acesso remoto, sem contato e obtendo a informação de seu conteúdo, assim, permitindo que todos os objetos físicos possa agir como nós em um mundo de rede física”. Mesmo nos dias de hoje, tais desafios continuam pertinentes [Krotov 2008].

O RFID tem a capacidade de transformar objetos inanimados em dispositivos inteligentes capazes de observar o mundo e manipulá-lo cada vez mais utilizando informações pessoais de determinado indivíduo [O’Connor 2005]. Segundo a Accenture [Accenture 2002], a interação inteligente entre objetos, sejam pessoas, animais ou mesmo itens do dia-a-dia, é potencialmente tão revolucionária quanto a Internet e a World Wide Web.

Quando se observa as possibilidades de aplicações utilizando RFIDs, tem-se a magnitude de abrangência desta tecnologia. Desde aplicações voltadas para saúde (cuidados com pessoas idosas, por exemplo) e assistência médica remota, monitoramento ambiental, serviços eletrônicos governamentais, determinação de localização e formação de redes sociais, monitoramento de desmatamentos, detecção de focos de contaminação, melhoria da logística de transportes urbanos e comerciais, monitoramento de transporte de alimentos, sensoriamento de bacias hidrográficas, pagamentos via dispositivos mobile, acesso fácil a serviços disponibilizados na web, etc. A mobilidade dos dispositivos e o acesso a diferentes serviços "anywhere/anytime" 
poderão vir a facilitar a inclusão digital de indivíduos de diferentes classes sociais fazendo com que o acesso à informação seja ampliado e diversificado. Assim, além de casas e edifícios inteligentes existentes hoje em dia pode-se verificar que inicia uma nova etapa neste processo, o de vilarejos e cidades inteligentes [Lee 2010]. Com isso, a tecnologia RFID (auxiliado por diversas tecnologias de sensoriamento, transmissão de dados sem fio, miniaturização de hardwares e maior poder de processamento na ponta da cadeia) pode permitir uma maior iteração entre objetos do mundo real com o mundo virtual, proporcionando uma comunicação fácil, simples e segura entre tais objetos [Ahson 2008] e isto se dá o nome de Internet das Coisas [INFSO 2008].

Com a Internet das Coisas começando a se tornar realidade pode-se visualizar um futuro onde os ambientes, lugares e até mesmo a cidade se tornará inteligente. Quando os objetos de uma cidade estiverem interligados através de sensores, os serviços públicos cada vez mais disponibilizados na cloud computing [Vaquero 2009] e o uso efetivo da TIC serão possíveis visualizar uma cidade inteligente através da disponibilização de diversos serviços, como o monitoramento do consumo de energia elétrica visando evitar desperdícios; monitoramento dos sistemas de água e barragens buscando comunicar a população quando alguma catástrofe está para acontecer devido às chuvas em um determinado período do ano; monitoramento de estradas, túneis, pontes e ferrovias como forma de identificar possíveis problemas e agir antecipadamente além de trazer informação em tempo real para a população; monitoramento de oleodutos e gasodutos visando controlar o consumo e evitar acidentes; dentre diversas outras possibilidades visando tornar a cidade com maior capacidade de inteligência sobre seus insumos.

\section{Um Framework para Cidades Inteligentes}

Baseado nas necessidades supracitadas se faz necessário facilitar o acesso e troca de informações entre serviços comuns e pessoas interessadas nestes serviços. Todavia, atualmente existe uma complexidade de diferentes tecnologias que visam auxiliar na construção de uma cidade inteligente.

Com isso, visando abstrair a complexidade tecnológica e facilitar a integração e desenvolvimento de serviços/aplicações para que os ambientes e/ou cidades possam se tornar mais inteligentes, este trabalho propõem uma plataforma para cidades inteligentes baseado na internet das coisas. A Figura 1 ilustra a plataforma proposta, onde pode ser dividido em:

- Camada física: composta por diversos equipamentos de hardware onde cada um tem sua especificidade e forma de comunicação. O objetivo desta camada é abstrair a forma de comunicação com estes dispositivos visando facilitar o desenvolvimento de aplicações. Por exemplo, controladores para automação predial/residencial utilizam protocolos como BACNet ${ }^{1}$ e Modbus ${ }^{2}$, os quais não são de amplo conhecimento de desenvolvedores de aplicações;

- Camada de dados: composta de um middleware que é capaz de acessar as informações da camada física, processar os dados, realizar filtros para tratamento dos dados e disponibilizar informações de fácil manipulação para a

\footnotetext{
${ }^{1}$ http://www.bacnet.org/

2 http://www.modbus.org/
} 
camada de serviços. Por exemplo, readers RFID realizam a leitura do ID de uma tag RFID inúmeras vezes enquanto ela permanecer no alcance da leitura e isto deve ser tratado corretamente para que a camada de serviços não realize $\mathrm{N}$ processamentos de uma função indevidamente;

- Camada de serviços: composta por duas API’s (Application Programming Interface) que irão disponibilizar diversos serviços para serem consumidos por aplicações na plataforma web e na plataforma mobile. Estas API’s irão facilitar o uso dos serviços padronizando a forma de acesso através de protocolos utilizados no mercado. Por exemplo: as redes sociais disponibilizam API's utilizando tecnologia de comunicação $\mathrm{JSON}^{3}, \mathrm{OAUTH}^{4}, \mathrm{OGP}^{5}$, etc; já o governo disponibiliza suas informações através de Web-Services/XML; por outro lado os meios de pagamento utilizam componentes desenvolvidos internamente.

- Camada da aplicação: onde aplicações de exemplo e tutorial serão disponibilizados em domínios diversos visando facilitar o entendimento e uso da plataforma em questão. Atualmente já existem duas aplicações de exemplo de uso (maiores detalhes serão apresentados na seção seguinte): (i) automação de iluminação e ar-condicionado via sensores RFID; (ii) monitoramento e controle do consumo de energia elétrica via sensores ZigBee.

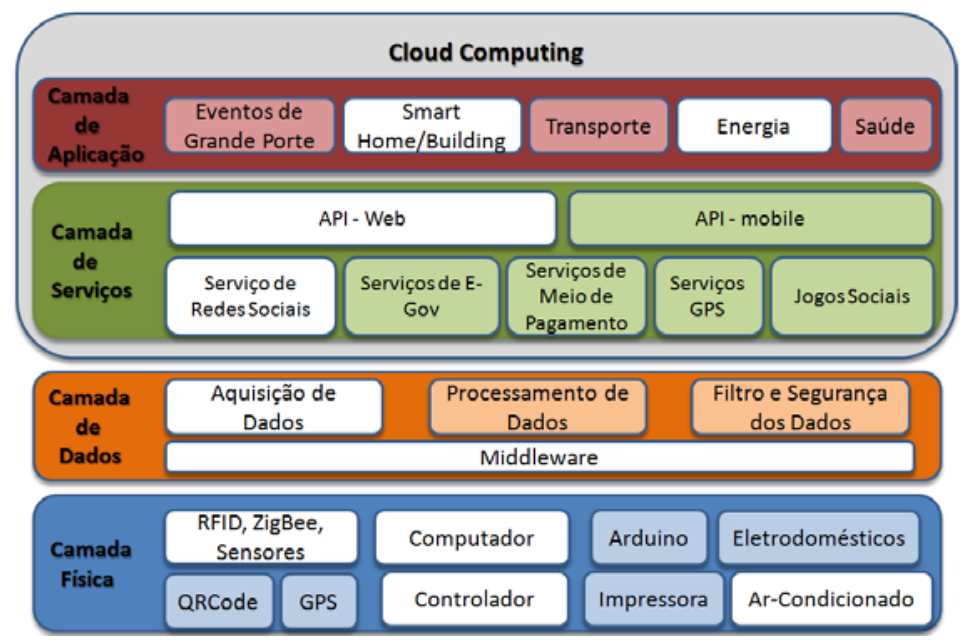

Figura 1. Plataforma para Cidades Inteligentes baseado na Internet das Coisas.

Atualmente, as caixas demarcadas com a cor branca já foram parcialmente ou completamente desenvolvidas no DComp da UFSCar - Sorocaba em parceria com o FIT/Flextronics (centro de excelência em RFID da América Latina) como forma de obter os primeiros protótipos de uso da plataforma em questão. Ainda, se faz importante ressaltar que as camadas de serviço e aplicação estão hospedadas na Cloud Computing do Google ${ }^{6}$ como forma de tornar os serviços amplamente acessíveis, flexíveis e escaláveis.

\footnotetext{
${ }^{3}$ http://www.json.org/

${ }^{4}$ http://oauth.net/

${ }^{5}$ http://ogp.me/

${ }^{6}$ https://appengine.google.com/
} 


\section{Casos de Uso Prático da Plataforma}

Visando validar a proposta da plataforma em questão foram desenvolvidos dois protótipos, os quais serão brevemente descritos a seguir:

\subsection{Automação de iluminação e ar-condicionado via sensores RFID}

Problema: Atualmente os hardwares para automação predial/residencial são programados através de circuitos digitais nos controladores, como por exemplo, o FX70 ${ }^{7}$ da Johnson Controls. Para que possa ser feito ajustes na temperatura de um ambiente automatizado é necessário que um técnico especializado esteja disponível durante um evento, por exemplo, para efetuar tais ajustes.

Solução: Para facilitar o controle da temperatura foi desenvolvida uma solução aonde as pessoas que irão para um evento, por exemplo, receberão tags RFID e informarão suas preferências de temperatura. Estas informações ficam armazenadas na tag RFID e durante o evento o reader RFID obtém as preferências das pessoas, calcula a média da temperatura desejada e é capaz de ajustar automaticamente a temperatura através da

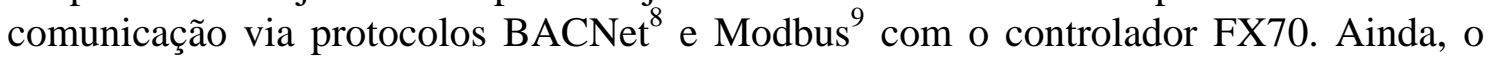
protótipo é capaz de identificar a quantidade de pessoas no evento e, caso contenha poucas pessoas, é capaz de desligar alguns ares-condicionados como forma de evitar desperdício de energia. Assim, os ambientes se tornam mais eficientes energeticamente e sustentáveis. Este projeto foi realizado em parceria com a empresa BrainSet ${ }^{10}$.

\subsection{Monitoramento e controle do consumo de energia elétrica via sensores ZigBee}

Problema: O monitoramento do consumo de energia e a disponibilização desta informação para os interessados fazem com que o consumo seja reduzido, simplesmente pelo fato de conhecer a informação. $\mathrm{O}$ uso deste tipo de monitoramento em alguns lares nos EUA reduziu o consumo de energia mensal de 20\% a 40\% [GooglePWS 2011].

Solução: Foi feita a aquisição de aparelhos P3, que são capazes de fazer a coleta dos dados de consumo de energia do ponto de energia (i.e. tomada elétrica). O envio destas informações para uma estação base foi feito por meio da tecnologia ZigBee, através da soldagem de uma antena de transmissão ZigBee no dispositivo de coleta P3 (Figura 2).

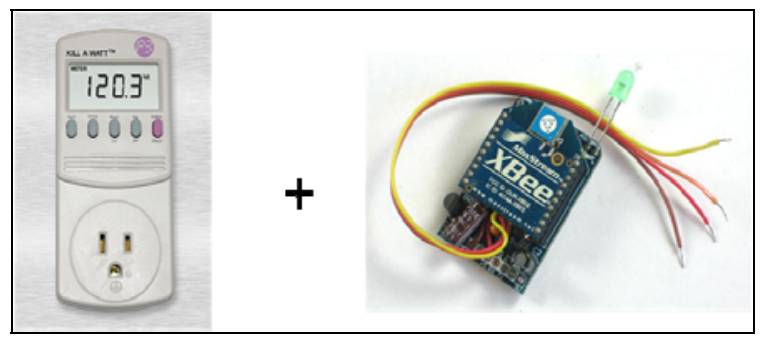

Figura 2. Dispositivo P3 com o transmissor ZigBee.

A coleta de dados na estação base foi realizada utilizando um script na linguagem Python e os dados foram inseridos em um banco de dados local, e em um banco de dados na nuvem, no Google App Engine. Assim o consumidor poderia acessar os dados

\footnotetext{
${ }^{7}$ http://bit.ly/wqmivh

8 http://www.bacnet.org/

9 http://www.modbus.org/

${ }^{10}$ http://www.brainset.com.br/
} 
de consumo através de seu dispositivo móvel, localmente através de Bluetooth e remotamente através da aplicação armazenada na nuvem.

\section{Conclusão e Trabalhos Futuros}

Este artigo apresentou uma plataforma para cidades inteligentes baseada na internet das coisas. O objetivo principal é disponibilizar uma plataforma que visa abstrair as complexidades de diversas tecnologias e protocolos de comunicação existente padronizando a forma de acesso e uso dos diversos hardwares, sensores, equipamentos e serviços existentes na web.

Como trabalhos futuros esperam-se o desenvolvimento de todos os módulos da plataforma e, assim, buscar parceiros no mercado para realizar o desenvolvimento de aplicações reais. Ainda, com a realização da Copa do Mundo e das Olimpíadas no Brasil, diversas oportunidades podem ser visualizadas com esta plataforma como: automação de hotéis, logística de transporte público, monitoramento e interação com os estádios, fácil acesso às informações turísticas no Brasil, dentre outras possibilidades.

\section{Agradecimentos}

Este trabalho foi apoiado parcialmente pelo Instituto Nacional de Ciência e Tecnologia para Engenharia de Software (INES) ${ }^{11}$, financiado pelo CNPq, processo 573964/2008-4.

\section{Referências Bibliográficas}

Pan, J.-G., Lin, Y.-F., Chuang, S.-Y., Kao Y.-C. (2011). "From Governance to Service- Smart City Evaluations in Taiwan”, In IEEE Conference on Service Sciences.

Ahson, S. and Ilyas, M. (2008) "RFID HANDBOOK: Application, Technology, Security and Privacy”. In CRC Press, Boca Raton, FL.

Vaquero, L. M., Rodero-Merino, L., Caceres, J., and Lindner, M. (2009). "A Break in the Clouds: Towards a Cloud Definition”. In SIGCOMM, 39(1):50-55.

Kehua, S., Jie, L., Hongbo, F. (2011) “Smart City and the Applications”, In: Internacional Conference on Eletronics, Communications and Controls (ICECC).

S. Sarma, D.L. Brock, K. Ashton, The networked physical world, Auto-ID Center, Disponível em: http://autoid.mit.edu/whitepapers/MIT-AUTOID-WH-001.PDF.

V. Krotov, I. Junglas, RFID as a Disruptive Innovation, Journal of Theoretical and Applied Electronic Commerce Research, Vol. 03, No. 02, 2008, pp. 44-59.

O’Connor, M.C. (2005). "RFID and the media revolution”, RFID Journal, Disponível em: http://www.rfidjournal.com/article/articleview/1508/1/1/. Dezembro, 2012.

Accenture. (2002). "Seize the day: The silent commerce imperative", Disponível em: http://www.accenture.com/NR/rdonlyres/1E69F408-F4D7-40E8-952F-

DAC9E60A0EA3/0/SeizeTheDay.pdf. Dezembro, 2012.

Lee, Y.W., Rho, S. (2010). "U-City Portal for Smart Ubiquitous Middleware”, In 12th ACM International Conference on Advanced Communication Technology (ICACT).

INFSO and EPoSS, (2008). "Internet of Things in 2020: A Roadmap for the Future”, Workshop Report, EC INFSO and EPoSS, Belgium.

GooglePWS, Google PowerMeter, (2011). http://www.google.com/powermeter/about/userstories.html, Dezembro.

\footnotetext{
${ }^{11}$ http://www.ines.org.br/
} 